\title{
Transcriptome analysis reveals salinity responses in four Tartary
} buckwheat cultivars

Jin-Nan Song, Xue-Hua Liu, Ya-Qi Wang, Hong-Bing Yang*

\section{Disclaimer Statement}

The authors have withdrawn this manuscript (BIORXIV/2019/869263) entitled Transcriptome analysis reveals salinity responses in four Tartary buckwheat cultivars because it requires adding new data and further modifications. Therefore, the authors do not wish this work to be cited as reference for the project. If you have any questions, please contact the corresponding author.

Hong-Bing Yang 